\title{
Even in correctable search, some types of rare targets are frequently missed
}

\author{
Michael J. Van Wert \\ Brigham and Women's Hospital, Boston, Massachusetts \\ AND \\ Todd S. Horowitz ANd Jeremy M. Wolfe \\ Brigham and Women's Hospital, Boston, Massachusetts \\ and Harvard Medical School, Boston, Massachusetts
}

\begin{abstract}
Socially important visual search tasks, such as airport baggage screening and tumor detection, place observers in situations where the targets are rare and the consequences of failed detection are substantial. Recent laboratory studies have demonstrated that low target prevalence yields substantially higher miss errors than do high-prevalence conditions, in which the same targets appear frequently (Wolfe, Horowitz, \& Kenner, 2005; Wolfe et al., 2007). Under some circumstances, this "prevalence effect" can be eliminated simply by allowing observers to correct their last response (Fleck \& Mitroff, 2007). However, in three experiments involving search of realistic X-ray luggage images, we found that the prevalence effect is eliminated neither by giving observers the choice to correct a previous response nor by requiring observers to confirm their responses. This prevalence effect, obtained when no trial-by-trial feedback was given, was smaller than the effect obtained when observers searched through the same stimuli but were given trial-by-trial feedback about accuracy. We suggest that low prevalence puts pressure on observers in any search task, and that the diverse symptoms of that pressure manifest themselves differently in different situations. In some relatively simple search tasks, misses may result from motor or response errors. In other, more complex tasks, shifts in decision criteria appear to be an important contributor.
\end{abstract}

On a regular basis, we must search for an object, such as a cell phone or a front door key that has been lost in the house. Or we might wish to locate that popular brand of cereal that seems to be hiding on the supermarket shelves amidst the sea of other options. In these and many other routine tasks, we can expect that the object of our search will be present most of the time; "target prevalence" is high. In contrast, in some very important visual search tasks, target prevalence is low. In airport security (Rubenstein, 2001) and medical screening tasks (Gur et al., 2004; Jiang, Miglioretti, Metz, \& Schmidt, 2007; Pisano et al., 2005; Smith \& Turnbull, 1997), for example, the targets (weapons, possible tumors) are very rare. Our desire to find these targets is high, but our expectation that one will be found in a specific stimulus is low.

Recent laboratory visual search experiments have shown that miss error rates are markedly elevated at low target prevalence. Wolfe, Horowitz, and Kenner (2005) reported that an artificial search task for tools among nontool distractors on a noisy background yielded far higher miss rates when targets were rare (.30 miss rate when targets appeared on $1 \%$ of trials) than when they were frequent (.07 miss rate when targets appeared on 50\% of trials). Moreover, correct target-absent reaction times
(RTs) were much faster at $1 \%$ target prevalence than at $50 \%$. Wolfe et al. (2007) replicated this "prevalence effect" using realistic X-ray airport baggage stimuli. They obtained a .46 miss rate at low prevalence $(2 \%$ target frequency) and a .20 miss rate at high prevalence $(50 \%$ target frequency). It is not known whether these effects are found in real-world settings with trained professionals and with grave consequences for errors. However, in light of the disquieting implications of these findings for real-world search contexts, the prevalence effect requires further scrutiny.

What mechanisms underlie the prevalence effect? Wolfe et al. (2007) argued that high miss error rates at low prevalence are not due to a simple speed-accuracy trade-off, but rather to a shift in decision criterion $(c)$, without a decline in sensitivity $\left(d^{\prime}\right)$ (Green \& Swets, 1966; Macmillan $\&$ Creelman, 2005). In fact, in Wolfe et al. (2007), $d^{\prime}$ was somewhat higher at low prevalence than at high prevalence. It appears that, when observers expect targets to be rare, they require less information to declare a bag free of weapons. This approach is beneficial for the majority of images, but it would increase the likelihood of declaring a target to be absent when a target is actually present. Similar shifts in criterion linked to changes in response

J. M.Wolfe, wolfe@search.bwh.harvard.edu 
probability have been demonstrated elsewhere in the signal detection literature (Healy \& Kubovy, 1981; Maddox, 2002). Offering a different account for the elevated miss error rates at low prevalence, Fleck and Mitroff (2007) attributed the prevalence effect to response errors caused by a bias to respond with the "target absent" key, even when a target was detected. Errors of this sort certainly occur in speeded, two-alternative forced choice (2AFC) tasks. Not infrequently, the observer wishes he could countermand a response already headed for the wrong finger. Such errors could be easily corrected by allowing observers to voluntarily reverse previous responses. Fleck and Mitroff gave observers this option in a replication of the original Wolfe et al. (2005) experiment. Without a correction option, they obtained a .27 miss error rate at $2 \%$ prevalence and a .08 miss error rate at $50 \%$ prevalence, demonstrating a significant prevalence effect. When provided with the opportunity to correct a response after a trial they deemed to be in error, Fleck and Mitroff's observers still produced a prevalence effect of standard magnitude before correction. However, after correction, the prevalence effect was essentially eliminated. A prevalence of $2 \%$ yielded a miss error rate of .10, and a prevalence of $50 \%$ yielded a miss error rate of .04. Fleck and Mitroff argued that the targetabsent response in low-prevalence blocks was "prepotent," liable to capture behavior even when the observer was trying to make the opposite response. More generally, even without explicit feedback, Fleck and Mitroff's observers seemed to know when they had made an error and could correct that error, if given the opportunity.

Note that motor errors are not the only route to correctable errors in experiments of this sort. Another type of error could be a form of speed-accuracy trade-off: Observers continue to search the stimulus or some memory of the stimulus after committing themselves to a "targetabsent" response. If they identify the target after commanding that "target-absent" response, they might know that an error had been made but be unable to countermand their response. Errors of this sort could also be eliminated by allowing observers to change answers after the fact.

Other attempts have been made to reduce response errors in low-prevalence visual search for nonluggage stimuli. Li, Li, Gao, Chen, and Lin (2006), using stimuli similar to those in the Wolfe et al. (2005) study, reported that the prevalence effect was reduced by changing the response procedure from a $2 \mathrm{AFC}$ task to a task in which the observer had to report the total number of targets in a block of trials. In our own work (Rich et al., 2008), we found that a prevalence effect could be obtained even with a very simple search for a $\mathrm{T}$ among $\mathrm{Ls}$, if the response was a $2 \mathrm{AFC}$ (e.g., $\mathrm{T}$ points left and, very rarely, right). However, when a $4 \mathrm{AFC}$ response was used (e.g., T points left, right, up, or, very rarely, down), the prevalence effect was eliminated. Like Fleck and Mitroff (2007), we argued that this version of the prevalence effect was produced by something like a prepotent motor response. In the $2 \mathrm{AFC}$ version, observers responded with one key on the overwhelming majority of trials and sometimes hit that key when they meant to hit the other, uncommon response key. In the $4 \mathrm{AFC}$ case, common responses were divided among three options, diluting the potency of any one response.

In more difficult search tasks, however, the prevalence effect has proved more stubborn. Fleck and Mitroff (2007) used stimuli similar to those in Wolfe et al. (2005) in their correction paradigm. In a subsequent study, we also attempted to eliminate errors due to overly hasty responses (Wolfe et al., 2007). This experiment used X-ray luggage stimuli in a closer visual approximation of the baggagescreening task. This is a more difficult search task; it produces false alarm errors and miss errors. False alarm errors decline at low prevalence, consistent with a criterion shift, as noted above. Using these stimuli, we found that forcing our observers to slow down did not eliminate the prevalence effect.

Another important factor is the role of feedback. In our previous work, we provided trial-by-trial feedback about accuracy. Fleck and Mitroff (2007) replicated that result without providing feedback to the observers. The prevalence effect they obtained, however, was eliminated when observers were allowed to correct responses. Even without explicit feedback, Fleck and Mitroff's observers often knew when they had made an error, and they were able to correct it.

The most pressing practical question is whether a prevalence effect is likely to occur in socially important search tasks, such as medical screening and airport security. In those contexts, the search task is hard, feedback is unreliable, and task demands make motor/response errors extremely unlikely. No one at an airport checkpoint is going to say to himself, "Oh dear! I saw a gun in that bag, but I pushed the wrong button, so I will have to let it go." Thus, if prevalence errors are motor/response errors, they are probably not an important contribution to errors at the airport or in the radiology suite. Conversely, if one wants to argue that prevalence effects are a realworld problem, it must be possible to show such effects in a task without feedback and with an opportunity to correct errors. That is what we show in the present experiments. Following Fleck and Mitroff (2007), we eliminate feedback and allow for correction (in different ways in Experiments 1A and 2). We apply these methods to the search of X-ray images of baggage, as in Wolfe et al. (2007). We conducted three experiments implementing response correction paradigms, with the goal of eliminating miss errors. In Experiments 1A and 1B, we applied Fleck and Mitroff's voluntary correction system to our simulated luggage-screening task. In Experiment 3, we employed a somewhat different compulsory correction system, in which observers were required to respond twice to the same display. To anticipate our results, with these stimuli, the prevalence effect will be smaller without feedback. However, that effect will persist even when observers are allowed to correct their responses.

\section{EXPERIMENT 1A Voluntary Correction Paradigm}

If the prevalence effect is due to response errors when targets are rare, allowing observers to correct their re- 
sponses should equalize miss rates at low and high prevalence. Experiment $1 \mathrm{~A}$ tested this hypothesis by applying Fleck and Mitroff's (2007) paradigm to the simulated luggage stimuli of Wolfe et al. (2007).

\section{Method}

Observers. Sixteen adults (19-53 years of age, $M=32.3$, $S D=$ $10.8,9$ female) were recruited from the Boston area to participate. No observer reported a history of eye or muscle disorders. All were screened for color blindness and normal visual acuity. Informed consent was obtained from all observers, and each was paid $\$ 10 / \mathrm{h}$ for his/her time.

Stimuli and Procedure. Observers participated in a laboratorysimulated airport luggage-screening task (see Wolfe et al., 2007, for more details). Stimuli consisted of JPEG X-ray images of empty bags combined with X-ray images of a variety of nonweapon objects (e.g., clothes, toys, containers) and weapons (100 images of knives and 100 images of guns). All X-ray images were obtained from the Department of Homeland Security's Transportation Security Laboratory (see, e.g., Figure 1). Each bag had a set size of 3, 6, 12, or 18 total items, plus a number of clothing objects (which add a pale orange cast to the image but do not appear as distinct items). Objects were placed at random throughout the bags and could overlap. The bags and contents were sized realistically (e.g., sunglasses were larger than paperclips). In bags containing a target weapon, only a single target was ever present. Stimuli were generated and presented using MATLAB 7.5 and the Psychophysics Toolbox (Brainard, 1997; Pelli, 1997), running on Macintosh G4 computers. At a viewing distance of $57 \mathrm{~cm}$, bags varied in size from $9.5^{\circ}$ of visual angle in height $\times 16^{\circ}$ of visual angle in width to $20^{\circ} \times 21.5^{\circ}$. The resulting images and screening circumstances, although not perfect, are valid approximations of those presented to security personnel at the airport.

Before the screening task, observers were shown 20 examples of potential target weapons for $1 \mathrm{sec}$ each, isolated from the bags, and were told to expect weapons to appear at a variety of angles and perspectives. Each trial began with a fixation cross and an audible click, followed $200 \mathrm{msec}$ later by the appearance of the stimulus, which remained visible until the observer responded. A 500-msec blank interval preceded the start of the next trial. Observers were instructed to indicate as quickly and accurately as possible whether a target was present or absent; they pressed one key for target present and a different key for target absent. We emphasized that finding weapons was the most important goal of the task, but we instructed observ-

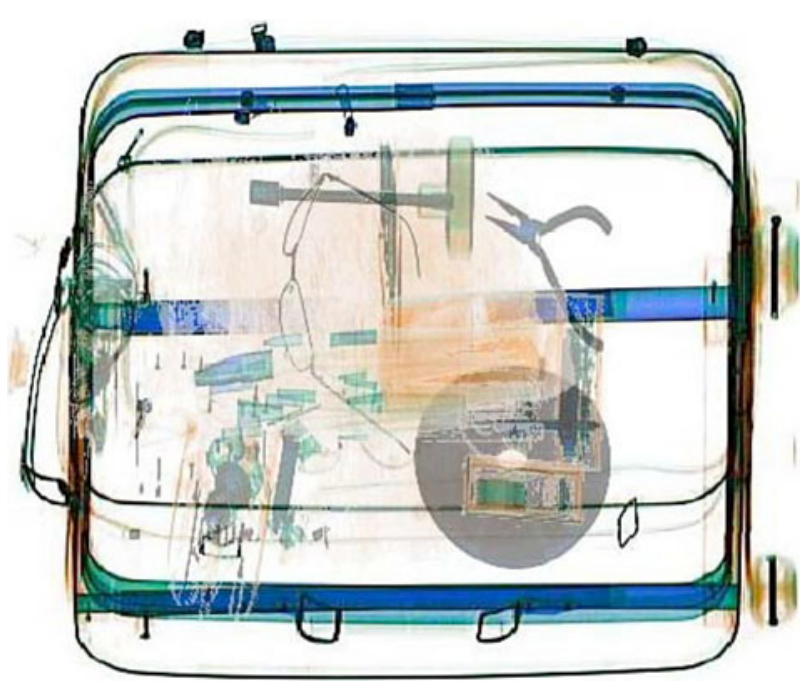

Figure 1. Sample bag display, set size 12, target absent. ers to keep in mind the real-world need to move people through the security checkpoint quickly.

As in Fleck and Mitroff (2007), observers had the option of correcting a response to the previous trial. Observers responded to trial $n$ and, following the 500-msec blank intertrial interval, the display for trial $n+1$ was presented. At this point, with the display for trial $n$ no longer visible, the observer had the option of reversing the response to trial $n$ by pressing the ESC key on the keyboard (i.e., if the initial response was "target absent," the ESC key would change it to "target present," and vice versa). Upon hitting the ESC key, observers would see a message saying, "You have reversed the prior response, now respond to this trial," and could then respond to the currently visible trial $n+1$. To be clear, observers could only reverse the previous response, not reexamine the previous display. No feedback about response accuracy was given at any point during the trials (before or after correction). Note that Fleck and Mitroff also ran a condition in which observers did not have the option of correcting previous responses. However, since Fleck and Mitroff had found that miss rates for observers who had not been given a correction option were comparable to miss rates prior to correction for those with the correction option, we decided that a no-correction condition would be redundant.

Each observer was given 100 practice trials at $50 \%$ prevalence (yielding 50 target-present trials) and was then tested on 200 experimental trials at $50 \%$ prevalence (100 target-present trials) and 1,000 experimental trials at $2 \%$ prevalence ( 20 target-present trials). Experimental-block order was counterbalanced across observers. Prior to each block, observers were informed about the rarity of targets: They were told that for high prevalence, targets would be "frequent," and for low prevalence, targets would be "rare." A 2-min break was enforced every 200 trials.

\section{Results}

In all experiments, we removed all RTs that were greater than $10,000 \mathrm{msec}$ or less than $200 \mathrm{msec}$. Error rates were arcsine transformed before analysis; we report the backtransformed means. ANOVAs and $t$ tests were conducted in SPSS 11 for Mac OS X. We report partial eta squared $\left(\eta_{\mathrm{p}}^{2}\right)$ as a measure of effect size for ANOVAs including factors of prevalence $(50 \%$ or $2 \%$ ), correction (before or after), and experimental-block order ( $50 \%$ or $2 \%$ first), unless otherwise specified. Levene's test of equality of error variances was used to determine equality of variance (for between-subjects comparisons), and corrections were made for statistical reporting, where appropriate. After RT filtering, only $1.1 \%$ of the trials were removed from Experiment 1A. Analyses were collapsed across set size, due to the sparse number of target-present trials at each set size at low prevalence.

The central finding of interest is that the correction paradigm failed to eliminate the prevalence effect (Figure 2). Low prevalence produced more miss errors than did high prevalence $\left[F(1,14)=17.908, p<.01, \eta_{\mathrm{p}}^{2}=.56\right]$. Correction lowered miss errors reliably, albeit very modestly in practical terms, by about .02 at low prevalence and .01 at high prevalence $\left[F(1,14)=23.663, p<.001, \eta_{\mathrm{p}}^{2}=.63\right]$. Experimental-block order did not significantly affect miss rate $(F<2.0, p>.15)$. There were no significant interactions. Paired samples $t$ tests were used to confirm the prevalence effect. The high-prevalence miss rate before correction was .17, significantly lower than the .26 lowprevalence miss rate before correction $[t(15)=4.745, p<$ $.001]$. Of critical importance, the situation was essentially unchanged after correction. The high-prevalence miss rate after correction was .16, lower than the .24 low-prevalence 


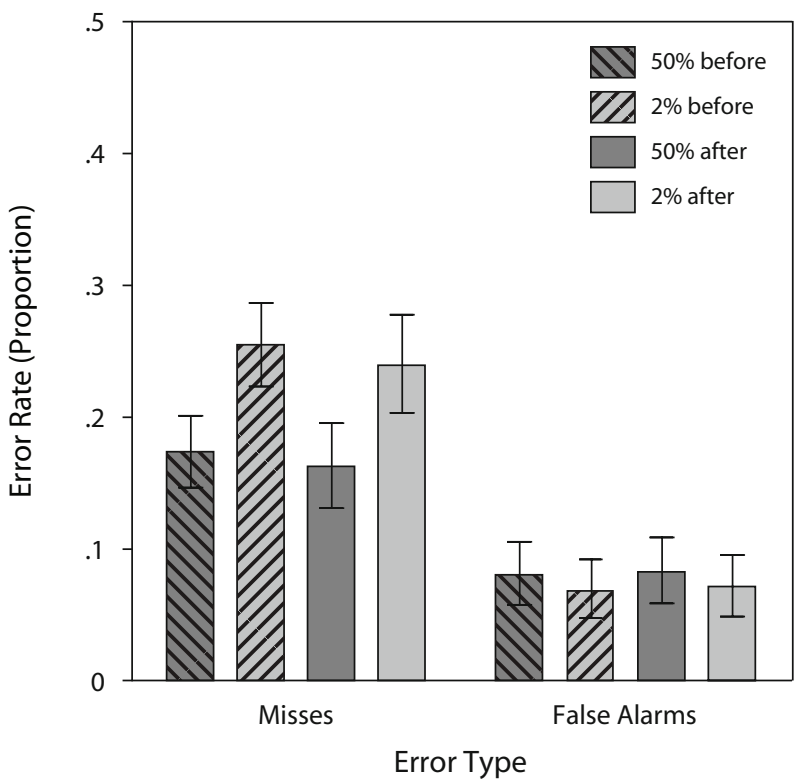

Figure 2. Mean error rates (misses and false alarms) for high $(50 \%)$ and low $(2 \%)$ prevalence. Error bars represent $95 \%$ within-subjects confidence intervals.

miss rate after correction $[t(15)=3.351, p<.01]$. Thus, a significant prevalence effect was present both before and after correction.

As was noted previously, the improvement with correction is marginal. At low prevalence, the numerically greatest number of changes were the 52 changes (out of 71 total changes) from correct "target-absent" responses to false alarm errors, although those 52 constitute a tiny fraction of the over 14,000 target-absent trials. Just $8(9.2 \%)$ of the 87 low-prevalence misses were corrected to hits. At high prevalence, $17(5.9 \%)$ of the 287 misses were corrected. Only $5(0.4 \%)$ correct "target-absent" responses became false alarms after correction.
Although Wolfe et al. (2007) found higher false alarms at high prevalence than at low prevalence, the present experiment did not show such a difference $(F<0.60, p>$ .40). This could be due to the fact that Wolfe et al. (2007) provided response feedback, whereas the present experiment presents no feedback (and, thus, more uncertainty), potentially leading to more false alarms at low prevalence. Correction increased the false alarm rate $[F(1,14)=6.946$, $p<.05, \eta_{\mathrm{p}}^{2}=.33$ ], but experimental-block order did not have any such effect $(F<0.001, p>.95)$. There were no significant two- or three-way interactions.

Wolfe et al. (2007) found that the prevalence effect manifested as a criterion shift. Figure 3 shows a similar result in the present experiment. Criterion values were lower at high prevalence $[F(1,14)=7.146, p<.05$, $\left.\eta_{\mathrm{p}}^{2}=.34\right]$ and slightly lower after correction $[F(1,14)=$ $\left.29.774, p<.0001, \eta_{\mathrm{p}}^{2}=.68\right]$. There was no main effect of experimental-block order on criterion $(F<0.65, p>.40)$, but there was a significant two-way interaction between prevalence and experimental-block order $[F(1,14)=$ 4.666, $p<.05, \eta_{\mathrm{p}}^{2}=.25$ ], reflecting the fact that there was a reliable criterion shift when observers completed the high-prevalence block followed by the low-prevalence block, but not vice versa.

Wolfe et al. (2007) reported that sensitivity did not change as a function of prevalence. In this experiment, there was a modest, but reliable, decrease in $d^{\prime}$ at low prevalence (see Figure 3, right panel) $[F(1,14)=5.156, p<$ $\left..05, \eta_{\mathrm{p}}^{2}=.27\right]$ and a modest increase in $d^{\prime}$ after correction $\left[F(1,14)=10.579, p<.01, \eta_{\mathrm{p}}^{2}=.43\right]$. There was no main effect of experimental-block order $(F<0.90, p>.35)$. There was a significant two-way interaction between correction and experimental-block order $[F(1,14)=5.018$, $\left.p<.05, \eta_{\mathrm{p}}^{2}=.26\right]$, where correction increased $d^{\prime}$ only in observers who completed the low-prevalence block followed by the high-prevalence block, but not vice versa.

In order to confirm that task duration and issues of fatigue and sustained attention were not major confounds

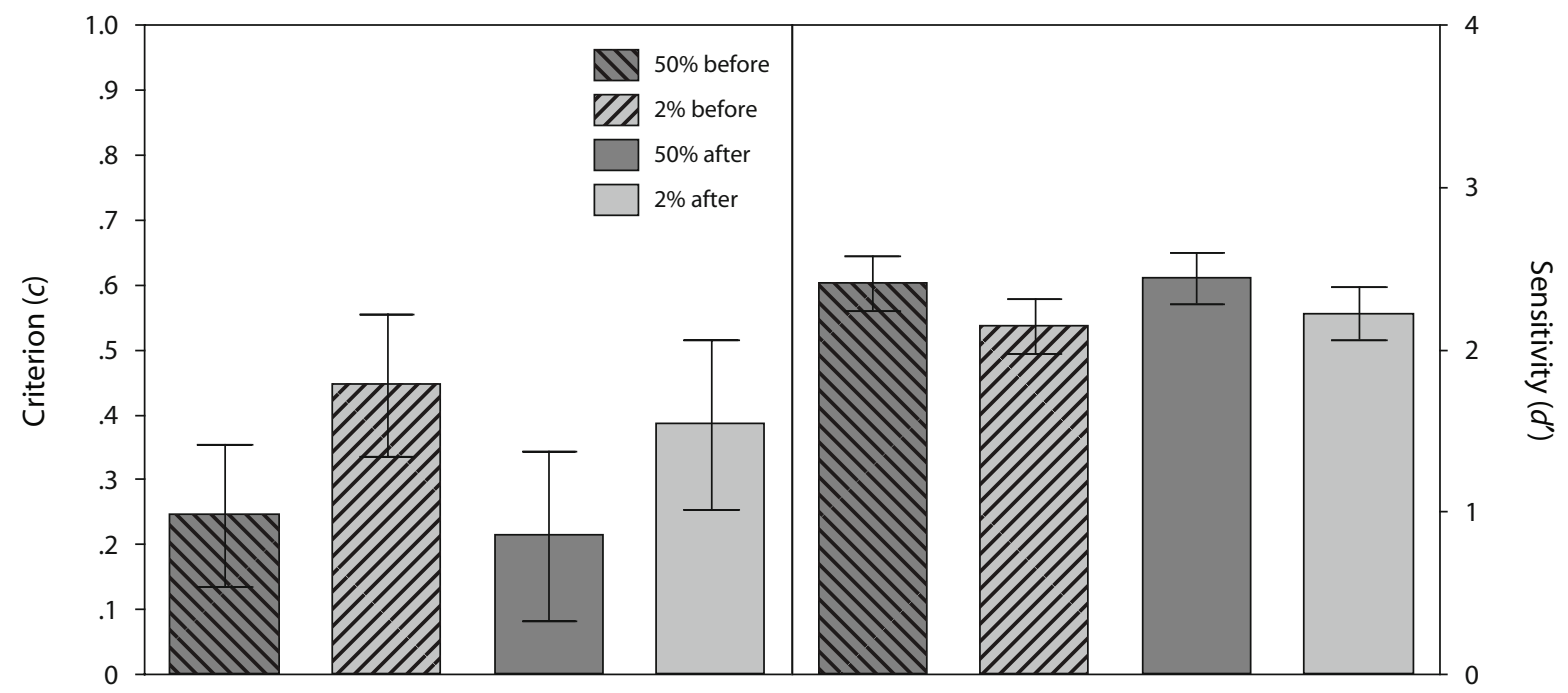

Figure 3. Mean criterion ( $c$, left panel) and sensitivity $\left(d^{\prime}\right.$, right panel) for high $(50 \%)$ and low $(2 \%)$ prevalence. Error bars represent $95 \%$ within-subjects confidence intervals. 
in our data, we broke down the low-prevalence portion of the task into 250-trial quartiles and analyzed whether miss error would increase significantly as the task progressed. Alertness could plausibly play some role in miss errors, but a repeated measures ANOVA on low-prevalence miss rate with the factors of quartile, correction, and experimentalblock order showed no reliable evidence that performance worsened as the task progressed $(F<2.7, p>.05)$. There were also no significant interactions. Of course, there could have been an effect too small for this analysis to detect, but it would have to have been quite small. Moreover, Wolfe et al. (2007) showed that a vigilance task inserted at various times throughout a low-prevalence block produced RTs that remained constant from beginning to end, suggesting that time-on-task was not the main cause of errors.

In the vigilance literature (Baddeley \& Colquhoun, 1969; Colquhoun \& Baddeley, 1967; Mackworth \& Taylor, 1963), the prevalence effect certainly has some important similarities to target frequency effects. However, as discussed more extensively in Wolfe et al. (2007), prevalence effects are not just vigilance effects by another name, as shown by the failure to find an effect of time-on-task in the present experiments.

Turning to RT data for before-correction responses only (after-correction RTs are not useful, due to a programming error), there was the usual main effect of response type ("yes" or "no"), demonstrating that "yes" responses were faster than "no" responses $[F(1,14)=26.355, p<$ $\left..001, \eta_{\mathrm{p}}^{2}=.65\right]$. When target-present and target-absent trials were taken together, there was no main effect of prevalence $(F<4.0, p>.05)$ or of experimental-block order $(F<0.10, p>.75)$. However, as is shown in Figure 4 , there was an interaction between prevalence and response type. This replicates Wolfe et al.'s (2005) find- ing that "no" responses are faster than "yes" responses at low prevalence, but we found the opposite to be true at high prevalence $\left[F(1,14)=69.656, p<.000001, \eta_{\mathrm{p}}^{2}=\right.$ .83]. Moreover, there was an interaction between response type and experimental-block order, showing that "yes" responses were faster than "no" responses, but only in the group that did the task in the low-prevalence condition first, followed by that in the high-prevalence condition $\left[F(1,14)=9.306, p<.01, \eta_{\mathrm{p}}^{2}=.40\right]$.

Dividing the RTs into the four possible response types (hit, correct target-absent, miss, false alarm), we found no main effect of prevalence $(F<0.10, p>.75)$ or of experimental-block order $(F<0.25, p>.65)$, but there was a significant effect of response type $[F(2.36,39)=$ $\left.39.109, p<.001, \eta_{\mathrm{p}}^{2}=.75\right]$. Prevalence and experimentalblock order interacted marginally $[F(1,13)=4.634, p=$ $\left..051, \eta_{\mathrm{p}}^{2}=.26\right]$, demonstrating that RTs in the highprevalence condition were faster than those in the lowprevalence condition, but only in the group that completed the low-prevalence block first. Prevalence also interacted with response type, indicating that hit RTs for low-prevalence targets were slower than those for highprevalence ones, but RTs were comparable at both prevalence levels for the other three response types $[F(2.45$, $\left.39)=4.175, p<.05, \eta_{\mathrm{p}}^{2}=.24\right]$. Finally, low-prevalence correct "target-absent" and "miss" responses were comparable $(t<1.2, p>.25)$.

In our previous studies, where observers received feedback after each trial, RT was slowed after miss errors. In the present study, observers did not receive feedback. However, if they committed an error and realized that they had committed that error, we might expect slowing on the next trial. Figure 5 shows average before-correction RTs for five trials before and after target-present trials in the low-prevalence condition (where essentially all of the sur-

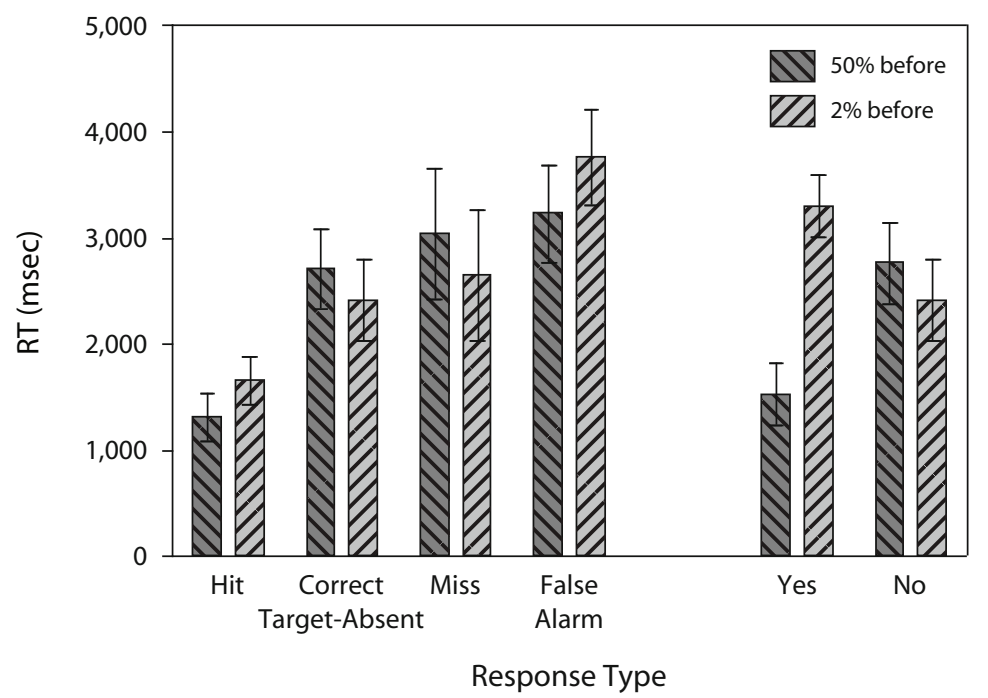

Figure 4. Reaction time (RT, in milliseconds), broken down by response type and prevalence for before-correction performance. On the left, RT is broken down by target presence and response, whereas on the right, RT is broken down only by participant response. Error bars represent $95 \%$ within-subjects confidence intervals. 


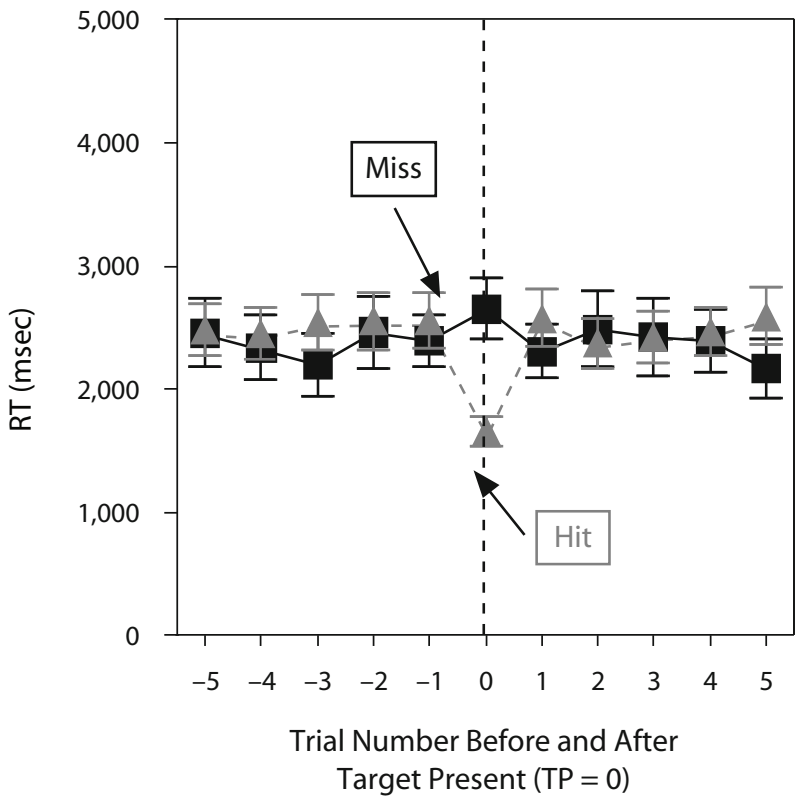

Figure 5. Low-prevalence reaction times (RTs, in milliseconds) before and after misses (black) and hits (gray) for trials where observers did not correct responses. Negative numbers on the $x$ axis denote trials preceding a target-present trial (0), and positive numbers indicate trials following the target. Error bars represent standard errors of the means.

rounding trials were target-absent trials). It is clear that there was no slowing after a miss trial $(F<0.01, p>.90)$. As noted above, the majority of errors were not corrected, so these RT data reflect the fact that observers were, on the whole, not cognizant of their mistakes. RTs for trials prior to a miss were, on average, $150 \mathrm{msec}$ faster than RTs for trials preceding hits, but this difference was not significant $(F<2.1, p>.15)$. The deviant RT in Figure 5 is the "hit" $\mathrm{RT}$, and that merely reflects that fact that target-present RTs were generally faster than target-absent ones.

\section{Discussion}

In Experiment 1A, the option to correct a previous response once a search display had disappeared did not eliminate the prevalence effect. As one might expect, when observers were offered the option of correcting a hasty, erroneous response, they did so and made some modest gains in accuracy/sensitivity. However, in the present experiment, a very small proportion of miss errors were corrected. Indeed, when targets were rare, the majority of reversals changed correct "target-absent" responses to false alarm errors. Observers' failure to correct miss errors in this task suggests that the prevalence effect in this experiment was not caused by response errors like those proposed by Fleck and Mitroff (2007) to account for their data. This does not make one result correct and the other result wrong. Instead, it supports the idea that prevalence effects manifest differently in different tasks. With relatively easy tasks - those that tend not to produce false alarm errors, as in Rich et al. (2008) and, perhaps, as in Fleck and Mitroff and Wolfe et al. (2005)-low- prevalence miss errors may well be due to motor errors, with observers making a speeded prepotent "no" response even when they know that the correct response is "yes." With the more difficult task used here, low prevalence manifests through a criterion shift.

Could it be that observers make more mistakes at low prevalence because they have less experience with the targets? Were this the case, observers who did the highprevalence block first should have shown less of a prevalence effect, but no reliable effect of this sort appears in the data.

It is useful to compare the present results with those from very similar experiments by Wolfe et al. (2007), who reported much larger criterion effects than we obtained here. For example, in Experiment 2 of that study, low prevalence shifted $c$ by about 1 unit (from about 1 at low prevalence to 0 for high prevalence). In the present experiment, the difference was only about .2 units (.45 for low prevalence and .25 for high). Correction did not alter this difference (.38 to .21$)$. We suspect that the lack of feedback was the critical variable.

Observers use feedback, explicit or implicit, to set decision criteria in search (Chun \& Wolfe, 1996). In tasks such as our baggage task, in the absence of explicit feedback, observers appear to move to a moderately conservative criterion in both the low- and high-prevalence conditions. Low prevalence still presses observers to a more conservative criterion, but not as dramatically. We will return to this topic in the General Discussion.

\section{EXPERIMENT 1B Replication With Target Preview}

With a realistic X-ray luggage screening task, Experiment $1 \mathrm{~A}$ demonstrated a prevalence effect that remained largely immune to correction. We did not have the proper experimental setup and statistical power to investigate the effect of prior experience on performance in Experiment 1A. Observers varied in their prior experience with this task, some of them having been in other experiments in the lab. Experiment 1B replicated Experiment 1A, with more control over observers' prior experience and more training with actual experiment targets.

\section{Method}

Observers. Ten adults (19-51 years of age, $M=27, S D=11$, 7 female) were recruited from the Boston area to participate. No observer reported a history of eye or muscle disorders, and all were screened for color blindness and normal visual acuity. Informed consent was obtained from all observers, and each was paid $\$ 10 / \mathrm{h}$ for his/her time. Important for present purposes, none of the observers had participated in a previous prevalence experiment in the laboratory.

Stimuli and Procedure. The stimuli and procedure in Experiment 1B were identical to those of Experiment 1A, with the following improvements. First, the observers had no prior experience with prevalence experiments in our lab. Second, observers were shown a slide show of the target guns and knives isolated on the screen for $2 \mathrm{sec}$ each. Finally, we made a minor improvement to the stimulus set by removing 28 target-present bag displays (out of the original 985) that contained targets deemed to be unrealistically positioned within the luggage. 


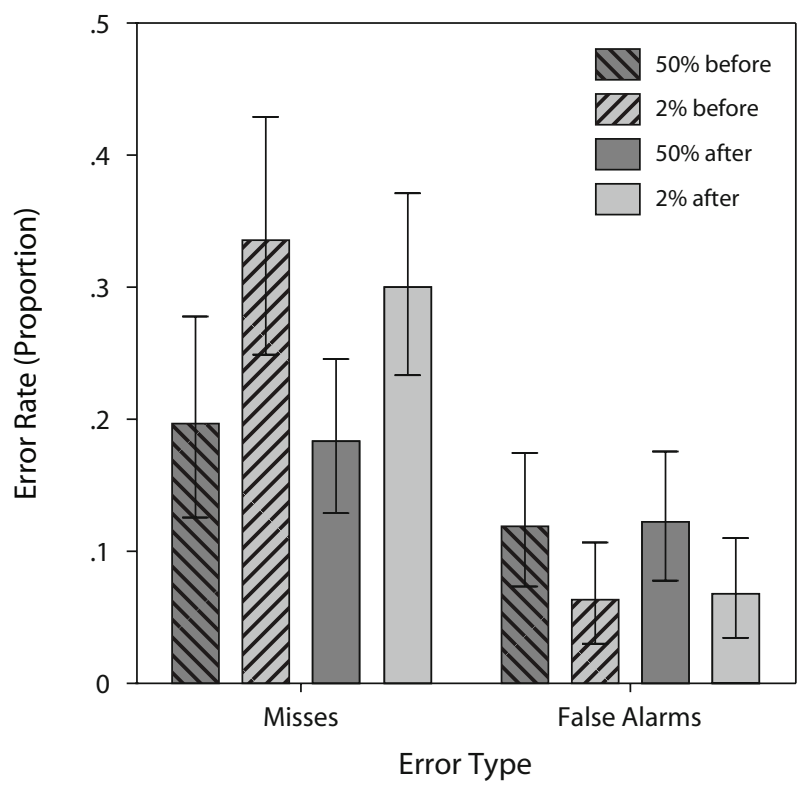

Figure 6. Mean error rates (misses and false alarms) for high $(50 \%)$ and low $(2 \%)$ prevalence. Error bars represent $95 \%$ within-subjects confidence intervals.

\section{Results and Discussion}

Error rates are shown in Figure 6. RT filtering and statistical analysis were the same as in Experiment 1A. RT filtering removed $3.6 \%$ of the trials, which were too long $(>10,000 \mathrm{msec})$.

The data replicate the prevalence effect demonstrated in Experiment 1A. Miss errors were significantly higher at low prevalence than at high prevalence $[F(1,8)=9.406$, $\left.p<.05, \eta_{\mathrm{p}}^{2}=.54\right]$, which was confirmed both before $[t(9)=2.588, p<.05]$ and after $[t(9)=2.875, p<.05]$ correction. Correcting a previous response also reliably reduced the miss rate $\left[F(1,8)=14.295, p<.01, \eta_{\mathrm{p}}^{2}=\right.$
.64]; but, as in Experiment 1A, this amounted only to a .02 reduction at high prevalence and about a .04 reduction at low prevalence. Similar to the results of Experiment $1 \mathrm{~A}$, only 7 out of the 67 misses $(10.5 \%)$ at low prevalence were reversed to a hit; 54 of the 79 total changes (68.4\%) reversed a correct "target-absent" response to a false alarm. There was no effect of experimental-block order $(F<0.60, p>.45)$, nor were there any significant interactions.

As in Experiment 1A, false alarm rates were not significantly different at low and high prevalence $(F<4.4, p>$ $.05)$, although the direction is consistent with prior results. False alarms decrease as miss errors increase. Correction $(F<1.5, p>.25)$ and experimental-block order $(F<$ $0.25, p>.65)$ did not affect false alarm rate. There were no significant interactions.

Once again, as is shown in Figure 7, the data support a criterion-shift account of the prevalence effect with these stimuli $\left[F(1,8)=9.376, p<.05, \eta_{\mathrm{p}}^{2}=.54\right]$. The prevalencerelated criterion shift manifests both before $[t(9)=2.734$, $p<.05]$ and after $[t(9)=2.833, p<.05]$ correction, even though criterion is more liberal after correction $[F(1,8)=$ $\left.8.793, p<.05, \eta_{\mathrm{p}}^{2}=.52\right]$. There was no main effect of experimental-block order $(F<0.05, p>.85)$, nor were there any significant two-way interactions. There was, however, a significant three-way interaction $[F(1,8)=5.519$, $\left.p<.05, \eta_{\mathrm{p}}^{2}=.41\right]$. Apparently, in the group that completed the high-prevalence condition first, correction shifted criterion more at low prevalence than at high prevalence, whereas, in the group that completed the low-prevalence condition first, correction shifted criterion more at high prevalence than at low prevalence. (Interpretation of this interaction is left as an exercise for the reader.)

Consistent with the findings of Wolfe et al. (2007), prevalence did not significantly affect sensitivity in this experiment $(F<0.10, p>.75)$. Corrections of previous responses, however, increased $d^{\prime}$ modestly but reliably

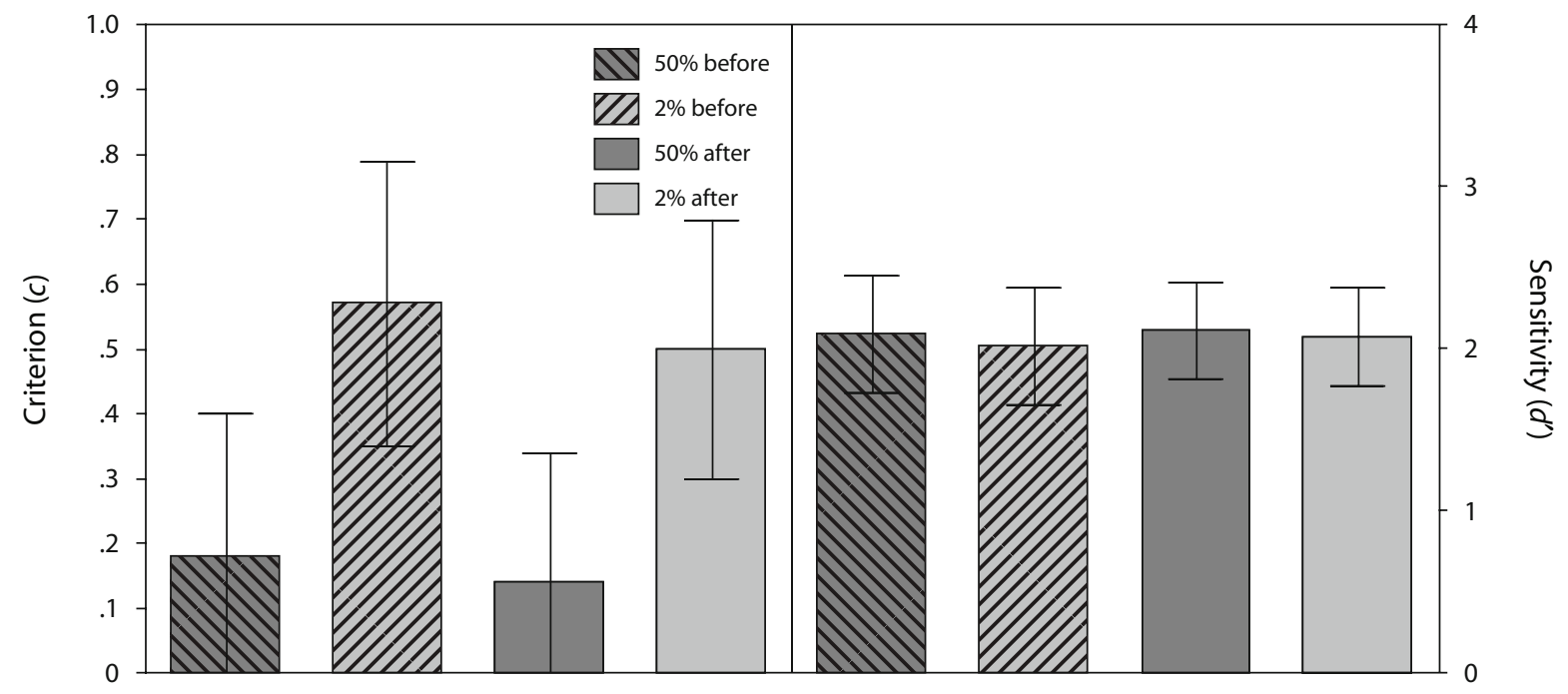

Figure 7. Criterion (c, left panel) and sensitivity $\left(d^{\prime}\right.$, right panel) for high (50\%) and low (2\%) prevalence. Error bars represent $95 \%$ within-subjects confidence intervals. 
$\left[F(1,8)=7.731, p<.05, \eta_{\mathrm{p}}^{2}=.49\right]$. Experimental-block order did not reliably affect sensitivity $(F<0.75, p>$ .40 ), and there were no interactions.

Checking once again to find out whether task duration is the primary cause of the higher miss rate at low prevalence, we analyzed the data in 250-trial quartiles in an ANOVA with factors of quartile, correction, and experimental-block order. As in Experiment 1A, miss rate did not increase reliably at low prevalence as the task progressed $(F<1.8, p>$ $.15)$. There were no significant interactions. The pattern of RT data was essentially the same as in Experiment $1 \mathrm{~A}$ and is omitted in the interest of space.

Experiment 1B replicated the results of Experiment 1A. This strengthens the argument that the prevalence effect in this task was not eliminated when observers were allowed to correct their answers. Preexposure to every target makes it less likely that the prevalence effect is due to unfamiliarity with the target items.

\section{EXPERIMENT 2 Compulsory Correction}

Given the very modest effects of allowing observers to voluntarily correct errors, it seemed advisable to force observers to make a second, confirming or correcting, response on every trial. We did this in Experiment 2.

\section{Method}

Observers. Sixteen adults (18-53 years of age, $M=33.6, S D=$ $11.1,8$ female) were recruited from the Boston area to participate. No observer reported a history of eye or muscle disorders, and all were screened for color blindness and normal visual acuity. Informed consent was obtained from all observers, and each was paid $\$ 10 / \mathrm{h}$ for his/her time. Ten of the observers in this experiment participated in Experiment 1A as well. The time between experiments ranged from 1 day to 1 month. Four observers did Experiment 1A first; six did Experiment 2 first.

Stimuli and Procedure. The stimuli, search task, and blocks were identical to those of Experiment 1A. Experiment 2, however, drew images from only one set of stimuli-2,012 possible target-absent and 989 target-present bag displays - whereas Experiment $1 \mathrm{~A}$ drew images from two stimulus sets. Half of the observers searched stimuli drawn from the set used in Experiment 2, and the other half searched through a different set of 2,015 target-absent and 985 target-present displays. There was no difference between the two sets, in terms of performance. In Experiment 2, observers were required to make two responses to each image display: an initial response and a confirmation. Once a display appeared, observers searched and then pressed either the "target present" or the "target absent" key on the keyboard. After the initial response, observers saw the message, "Please either confirm or change your response." Observers then pressed the same key as that used for their initial response if they wanted to confirm their first decision, or they pressed the other key if they wanted to change their original decision. Observers then saw the message, "Thanks," and moved on to the next trial. The search display remained visible during the whole response process, allowing observers to continue searching after the initial response. Observers received no response feedback during the task.

\section{Results and Discussion}

RT filtering and statistical analysis were the same as in Experiment 1A. RT filtering removed 2.2\% of trials, which were too long $(>10,000 \mathrm{msec})$. Unless specified otherwise, the ANOVAs included factors of prevalence, confirmation, and experimental-block order.

Error data are shown in Figure 8. There was a significant prevalence effect, as shown by the greater proportion of miss errors at low prevalence $[F(1,14)=8.378, p<$ $\left..05, \eta_{\mathrm{p}}^{2}=.37\right]$. There were fewer miss errors on the second response than on the first $[F(1,14)=13.902, p<.01$, $\left.\eta_{\mathrm{p}}^{2}=.50\right]$. Note that observers could continue to search the display during the interval between their first and second responses, so a reduction in miss errors is not particularly surprising. We find it important that even though the miss rates were reduced, a significant prevalence effect was seen in the second responses. The .23 miss rate on the second response in the low-prevalence condition was significantly higher than the .17 miss rate on the second response in the high-prevalence condition $[t(15)=2.219, p<.05]$. There was no main effect of experimental-block order $(F<0.01$, $p>.90)$, nor were there any significant interactions

As before, false alarms decreased as misses increased. There were more false alarms at high prevalence $[F(1,14)=$ $\left.7.734, p<.05, \eta_{\mathrm{p}}^{2}=.36\right]$ and more false alarms on the second response $\left[F(1,14)=14.414, p<.01, \eta_{\mathrm{p}}^{2}=.51\right]$. Experimental-block order, however, did not affect the false alarm rate $(F<0.60, p>.45)$. There was a reliable interaction between prevalence and confirmation, indicating that false alarm rates were higher on the second response only under low-prevalence conditions $[F(1,14)=7.382, p<$ $\left..05, \eta_{\mathrm{p}}^{2}=.35\right]$. Other interactions were not significant.

In this experiment, the requirement of confirming responses allowed observers to improve their overall accuracy by $9.4 \%$ at low prevalence $[t(15)=3.022, p<.01]$ and by $3 \%$ at high prevalence $[t(15)=3.337, p<.01]$. At low prevalence, because absent trials were so common, $81 \%$ of all 306 changes were from correct "target-absent"

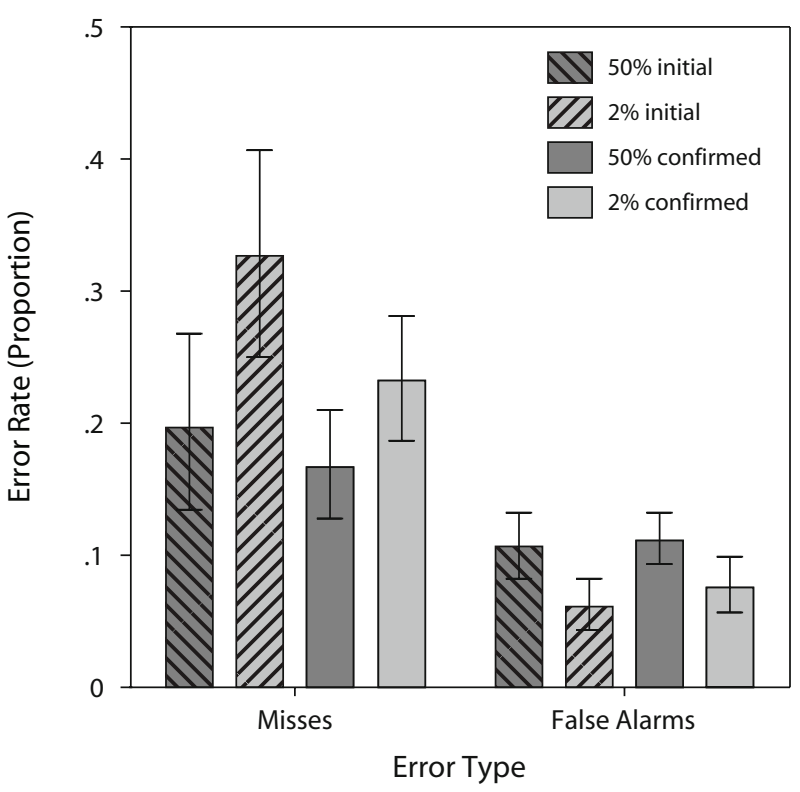

Figure 8. Mean error rates (misses and false alarms) for high $(50 \%)$ and low (2\%) prevalence. Error bars represent $95 \%$ within-subjects confidence intervals. 
Table 1

Percentages of First Responses

That Were Changed on Second Response

\begin{tabular}{|c|c|c|c|c|}
\hline Prevalence & Miss to Hit & $\begin{array}{c}\text { Correct } \\
\text { Absent to } \\
\text { False Alarm }\end{array}$ & Hit to Miss & $\begin{array}{c}\text { False Alarm } \\
\text { to Correct } \\
\text { Absent }\end{array}$ \\
\hline \multicolumn{5}{|l|}{ Experiment 1A } \\
\hline Low $(2 \%)$ & 9.2 & 0.4 & 0.0 & 9.7 \\
\hline High $(50 \%)$ & 5.9 & 0.4 & 0.0 & 6.8 \\
\hline \multicolumn{5}{|l|}{ Experiment 1B } \\
\hline Low $(2 \%)$ & 10.5 & 0.6 & 0.0 & 2.8 \\
\hline High $(50 \%)$ & 8.0 & 0.6 & 0.4 & 2.3 \\
\hline \multicolumn{5}{|l|}{ Experiment 2} \\
\hline Low $(2 \%)$ & 29.9 & 1.7 & 0.5 & 2.4 \\
\hline High $(50 \%)$ & 16.7 & 1.9 & 0.4 & 10.1 \\
\hline
\end{tabular}

Note-At low prevalence in Experiment 1A, only 9.2\% of the targets missed on first response were found on the second; in Experiment 1B, $10.5 \%$, and in Experiment 2, 29.9\%.

responses to false alarms. Note that these correct responses to false alarm changes represent only $1.7 \%$ of the target-absent trials; but because the vast majority of trials were target-absent trials, this $1.7 \%$ represents fully $81 \%$ of all changes. At high prevalence, however, the pattern was somewhat different, with $25 \%$ of the 104 changes being from correct "target-absent" responses to false alarms and $52 \%$ being from miss errors to hits. Table 1 breaks down each change by response type for Experiments 1A, 1B, and 2. The miss-to-hit percentages for Experiment 2 make it clear that requiring a second response allowed observers to correct about one third of their low-prevalence miss errors. Even if it did not eliminate the prevalence effect, the required response did narrow the gap between low- and high-prevalence conditions.

As is shown in Figure 9, analysis of the signal detection parameters $d^{\prime}$ and $c$ replicates the findings of Experiments $1 \mathrm{~A}$ and $1 \mathrm{~B}$, as well as those of Wolfe et al. (2007). Criterion was more conservative at low prevalence $\left[F(1,14)=9.589, p<.01, \eta_{\mathrm{p}}^{2}=.41\right]$ and more liberal after correction $\left[F(1,14)=18.294, p<.01, \eta_{\mathrm{p}}^{2}=.57\right]$.
Experimental-block order did not affect criterion $(F<$ $0.10, p>.75)$. There was an interaction between prevalence and confirmation, reflecting the larger effects of correction at low prevalence $[F(1,14)=5.732, p<.05$, $\left.\eta_{\mathrm{p}}^{2}=.29\right]$. Other interactions were not significant.

Turning to sensitivity, $d^{\prime}$ improved modestly on the second response $\left[F(1,14)=7.651, p<.05, \eta_{\mathrm{p}}^{2}=.35\right]$. However, there was no main effect of prevalence $(F<0.30$, $p>.55)$ or experimental-block order $(F<0.60, p>.45)$. There were no significant interactions.

As before, miss rate was analyzed in 250-trial quartiles in an ANOVA with factors of quartile, confirmation, and experimental-block order. Miss rate did not increase reliably as the task progressed $(F<0.10, p>.95)$. There was a significant interaction between confirmation and quartile $\left[F(3,42)=4.010, p<.05, \eta_{\mathrm{p}}^{2}=.22\right]$, reflecting the fact that the second response was associated with reduced miss rates only in the second half of the experiment. Other interactions were not significant.

Experiment 2 demonstrated that requiring observers to confirm their responses on every trial improved performance modestly. That improvement could have been due to the correction of perceived errors - as in Fleck and Mitroff (2007) - or it could have been the by-product of forcing observers to spend more time with each stimulus. Wolfe et al. (2007) found that forcing observers to take a second look at a stimulus on a few trials improved performance on those specific trials. These intermittent second looks were intended to induce a general slowing of first responses. This manipulation did slow first responses significantly, but it did not reduce the prevalence effect. Essentially the same point is made here. Performance is better on the second response than on the first response, but the prevalence effect remains.

Effect of removing feedback. The original purpose of these experiments was to examine the possibility that the prevalence effect could be eliminated by allowing observers to correct responses. The effects of correction in our

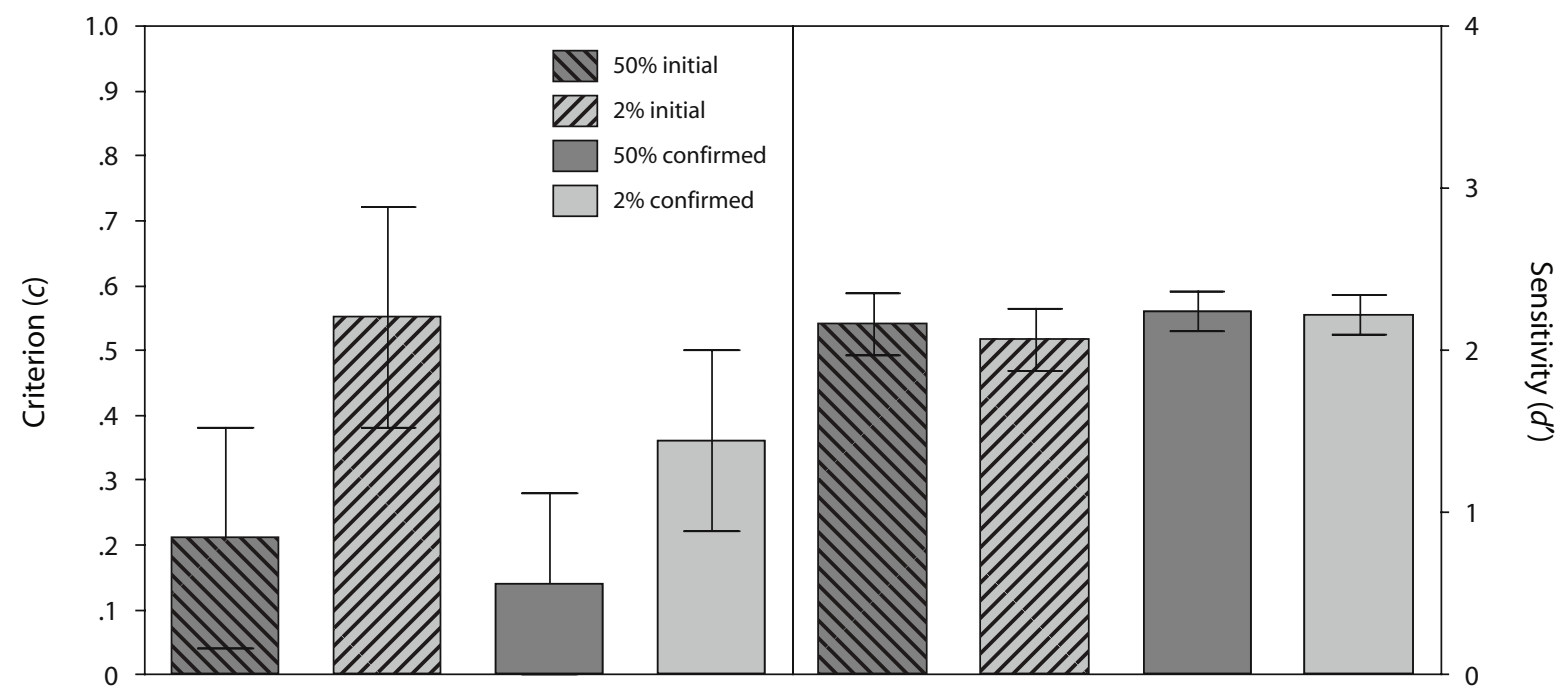

Figure 9. Criterion ( $c$, left panel) and sensitivity ( $d^{\prime}$, right panel) for high $(50 \%)$ and low $(2 \%)$ prevalence. Error bars represent $95 \%$ within-subjects confidence intervals. 
experiments were quite small. The biggest change from our earlier results (Wolfe et al., 2007) seems to be associated with the presence of feedback in our earlier experiments and its absence in these experiments. The prevalence effect, although still robust in the present experiments, appears smaller than that in comparable earlier experiments. In order to assess this hypothesized role of feedback, we did a post hoc comparison of Experiments 1A, 1B, and 2 from Wolfe et al. (2007) with Experiments 1A and 2 from the present study; Experiment 1B was omitted because of its redundancy with Experiment 1A. The tasks in these two sets of experiments were nearly identical, except that Wolfe et al. (2007) provided full feedback, and the present experiments provided none. As in the present study, each of the three Wolfe et al. (2007) experiments tested a strategy intended to eliminate the prevalence effect (e.g., combining search performance of pairs of observers, or forcing observers to slow down). Each strategy failed to eliminate the prevalence effect.

For analysis of RT performance, we compared data for uncorrected responses from the present Experiment 1A with data from Experiment 1A in Wolfe et al. (2007). It is possible that having the ability to correct responses in the present experiments could have influenced performance (RT, error rate) on subsequent initial responses. Nevertheless, this between-experiments comparison provides support for the hypothesis that feedback produced more dramatic shifts in criterion, and thus in errors, as a function of target prevalence.

Figure 10 shows the accuracy data from Experiments 1A and 2, plotted in $z$-coordinates. Each experiment is plotted as two barbells: one before correction (circles) and one after correction (diamonds). Each barbell consists of a symbol on the lower left end of the barbell, denoting performance at $2 \%$, and a symbol on the upper right end, denoting performance at $50 \%$. Similarly, the large oval summarizes performance data from comparable experiments in Wolfe et al. (2007) at $2 \%$, and the large rectangle summarizes 2007 performance at $50 \%$. The dotted line is the receiver operating characteristic (ROC) with a slope of 0.6 that best fits the Wolfe et al. (2007) data. Movement along the ROC reflects a criterion shift. The solid line (bottom right) is the diagonal line of unit slope on a standard ROC. Had the present experiments also yielded a criterion shift, the barbell shafts would be parallel to the ROC. In contrast, a sensitivity change would result in barbell shafts perpendicular to the ROC.

Several points can be made from this figure. The central conclusion is that, in the absence of feedback, the prevalence effect was smaller but was still characterized by a criterion shift. The data from the present studies (no feedback) fall roughly on the line in $z$-coordinate space defined by the data from the Wolfe et al. (2007) experiments (with feedback). This corresponds to an ROC with a slope of about 0.6. If the primary effect of the absence of feedback had been to change sensitivity, the barbells representing the prevalence effect would have been oriented orthogonally to this line.

Second, the without-feedback data points from the present experiments lie between the low- and high-prevalence points from the with-feedback conditions of Wolfe et al.

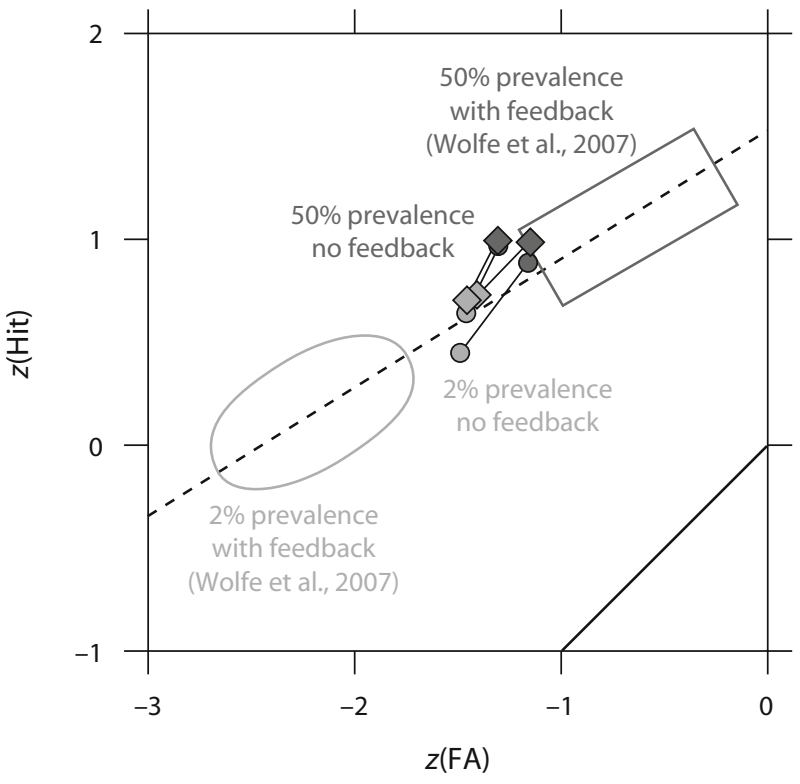

Figure 10. Data from $2 \%$ and $50 \%$ prevalence experiments with feedback (from Wolfe et al., 2007) and without feedback (present experiments). There is a greater shift in criterion with feedback than without. The dotted line represents a receiver operating characteristic of slope 0.6 in $z$-coordinates. The large open oval shows the range of $2 \%$ prevalence data from Figure 18 of Wolfe et al. (2007). The large open rectangle shows the $50 \%$ prevalence data from Wolfe et al. (2007). Data from Experiments 1 and 2 of the present article are shown as barbells between the open figures. In all barbells, the end to the lower left represents $2 \%$ prevalence. Ends to the upper right represent $50 \%$ prevalence. The steeper barbells are from Experiment 1. The circles show before-correction values, and the diamonds show after-correction values.

(2007). In the absence of explicit feedback, criteria at both low and high prevalence move to intermediate values. A mixed model ANOVA that includes only initial response data from the present Experiments 1A and 2, with factors of prevalence and feedback, reveals a main effect of feedback on criterion: Overall, criterion values are closer to zero in the absence of feedback $[F(1,76)=12.224, p<$ $\left..01, \eta_{\mathrm{p}}^{2}=.14\right]$. There is the usual effect of prevalence, such that the criterion is more neutral at high prevalence than at low prevalence $\left[F(1,76)=164.03, p<.00001, \eta_{\mathrm{p}}^{2}=\right.$ .68]. As is clear from Figure 10, the effects of prevalence (i.e., the distance between linked circles and diamonds) are much more marked with feedback than without $\left[F(1,76)=51.086, p<.00001, \eta_{\mathrm{p}}^{2}=.40\right]$.

Questioning the assumptions of $\boldsymbol{d}^{\prime}$ and $c$. As measures of sensitivity and criterion, $d^{\prime}$ and $c$ are based on the assumption of an equal variance $\mathrm{ROC}$ with a slope of 1.0. If we take the "true" slope to be 0.6, as shown in Figure 10, we can calculate the alternative measures $d_{\mathrm{a}}$ and $c_{\mathrm{a}}$ for Experiments 1A and 2 (see Macmillan \& Creelman, 2005 , chap. 3). Analyzed in this way, the results suggest some effect of prevalence on both criterion and sensitivity. The change in criterion with prevalence, as measured by $c_{\mathrm{a}}$, is significant for three of the four comparisons $[t(11)>$ $2.4, p<.05]$. The criterion shift for the second response in 
Table 2

Nonparametric Sensitivity $\left(A^{\prime}\right)$ and Bias $\left(B^{\prime \prime}\right)$ Values Calculated From Average Hit and False Alarm Data for Experiments 1 and 2

\begin{tabular}{lccccc}
\hline & \multicolumn{2}{c}{$A^{\prime}$} & & \multicolumn{2}{c}{$B^{\prime \prime}$} \\
\cline { 2 - 3 } \cline { 5 - 6 } & $\begin{array}{c}\text { Before } \\
\text { Correction }\end{array}$ & $\begin{array}{c}\text { After } \\
\text { Correction }\end{array}$ & & $\begin{array}{c}\text { Before } \\
\text { Correction }\end{array}$ & $\begin{array}{c}\text { After } \\
\text { Correction }\end{array}$ \\
\hline Experiment 1 & & & & & \\
2\% Prevalence & .90 & .91 & & .65 & .60 \\
50\% Prevalence & .92 & .92 & & .36 & .31 \\
Experiment 2 & & & & & .75 \\
2\% Prevalence & .88 & .91 & & .75 & .56 \\
$50 \%$ Prevalence & .90 & .91 & & .30 & .18 \\
\hline
\end{tabular}

Note-Maximum $A^{\prime}=1.0$. For $B^{\prime \prime}$, zero indicates no bias.

Experiment $1 \mathrm{~A}$ is marginal $[t(11)=2.1, p=.057]$. The change in sensitivity, as measured by $d_{\mathrm{a}}$, is significant in Experiment $1 \mathrm{~A}$ [both $t \mathrm{~s}(11)>3.1, p<.01$ ], but not in Experiment 2 (both $t \mathrm{~s}<1.7, p>.1$ ).

We can also measure $A^{\prime}$ (proposed as a nonparametric measure of the area under the ROC) and $B^{\prime \prime}$ (a measure of bias) (Donaldson, 1992). Average results are shown in Table 2. The nonparametric nature of these measures has been challenged (Pastore, Crawley, Berens, \& Skelly, 2003), but these measures also show the pattern of results described throughout this article: little or no effect of prevalence on sensitivity, with a substantial effect on criterion. Correction has more of an effect in Experiment 2 , as would be expected when all responses must be confirmed.

The absence of feedback also has a pronounced effect on RT. Observers appear to have adopted a more cautious approach to the task, spending more time with each display. This effect is illustrated in Figure 11. It is obvious that the main effect of feedback was on the "no target" responses; analyses were not done on false alarm responses because rates were very low at $2 \%$ prevalence for the Wolfe et al. (2007) study. These were much faster with feedback than without. A mixed model ANOVA on RT (collapsed across set size), with factors of feedback, prevalence, and response type, revealed main effects of feedback $\left[F(1,38)=17.040, p<.001, \eta_{\mathrm{p}}^{2}=.31\right]$, prevalence $\left[F(1,38)=5.991, p<.05, \eta_{\mathrm{p}}^{2}=.14\right]$, and response type $\left[F(2,76)=80.119, p<.001, \eta_{\mathrm{p}}^{2}=.68\right]$, as well as reliable interactions between response type and feedback $\left[F(2,76)=13.841, p<.001, \eta_{\mathrm{p}}^{2}=.27\right]$ and prevalence and response type $\left[F(2,76)=22.776, p<.001, \eta_{\mathrm{p}}^{2}=\right.$ .38]. Looking particularly at the RTs at low prevalence, absence of feedback slowed correct "target-absent" RT by $1,126 \mathrm{msec}[t(38)=5.043, p<.001]$, hit RT by $329 \mathrm{msec}$ $[t(38)=2.084, p<.05]$, and miss RT by $1,336 \mathrm{msec}$ $[t(38)=5.058, p<.001]$. A similar, but more modest, effect occurs at high prevalence: Correct "target-absent" RT slows by $682 \mathrm{msec}[t(38)=2.231, p<.05]$, hit RT by $258 \mathrm{msec}[t(20.9)=2.270, p<.05]$, and miss RT by $877 \mathrm{msec}[t(38)=2.411, p<.05]$. Thus, although there is still a persistent prevalence effect in the no-feedback experiments, the absence of feedback makes the observers more cautious and more accurate.

We find it interesting that Fleck and Mitroff (2007) did not find a major effect of feedback. They replicated the original Wolfe et al. (2005) "find the tools" experiment without feedback. Nevertheless, they obtained a substantial prevalence effect. It was this prevalence effect that was correctable in their experiment. We will discuss

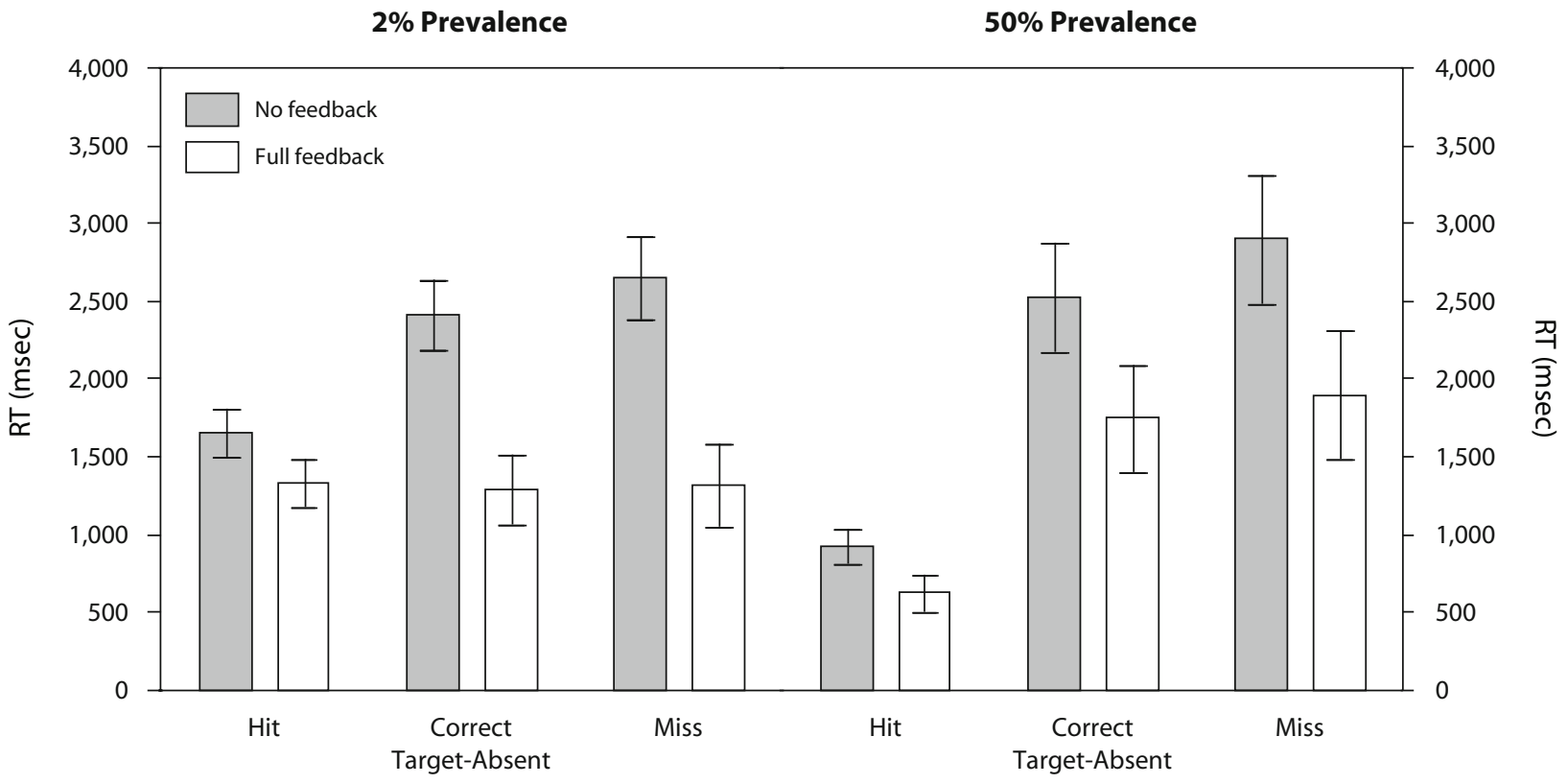

Response Type

Figure 11. RTs (in milliseconds) for correct target-absent, hit, and miss responses. Full feedback (Experiment 1A of Wolfe et al., 2007) is represented in white bars, and no feedback (initial response data from Experiment $1 \mathrm{~A}$ of the present study) is represented in gray bars. Error bars represent $95 \%$ between-subjects confidence intervals. 
this difference in the effects of feedback in the General Discussion.

\section{GENERAL DISCUSSION}

We believe that low prevalence puts pressure on the observer. This pressure can manifest in different ways, depending on the details of the search task. Fleck and Mitroff's (2007) observers responded to this pressure with speeded errors that they recognized and could correct. The observers in the present experiments responded to our somewhat different stimuli with a criterion shift that produced more miss errors and fewer false alarms. This pattern of errors was not eliminated by the opportunity to correct responses. The object-search tasks used in Wolfe et al. (2005) and by Fleck and Mitroff were fairly demanding, but they did not produce false alarm errors. It is a "high threshold" task (Palmer, Verghese, \& Pavel, 2000) that would occupy space in the upper left corner of a standard ROC graph (high hits with low false alarms). There would not be much room in this task for a criterion shift to manifest. In this part of the task space, many of the errors produced by low prevalence seemed to be errors that the observer noticed and was allowed to correct.

In the simulated baggage task used here, false alarms occurred, and $d^{\prime}$ hovered around 2.0. With this task, Wolfe et al. (2007) found that the main effect of prevalence was on criterion. In the present experiments, when feedback was eliminated, the effect of criterion was muted. Both low- and high-prevalence criteria moved toward an intermediate value. Nevertheless, we still observed a significant criterion shift due to prevalence.

Fleck and Mitroff (2007) produced a robust prevalence effect with their no-feedback task. We hypothesize that their task provided its own feedback: In tasks that did not produce false alarms, observers may have had to work to find the target; but upon finding it, they could be quite sure that they had indeed found it. Fleck and Mitroff's correction data show that their observers were often aware that they had found the target but had made the incorrect response. They could correct the error that they had detected. Our observers did not explicitly detect their errors and could not correct them with a second response.

Neither the Fleck and Mitroff (2007) results nor our present results represent the "true results." Rather, each samples a different portion of a task space. From a practical point of view, we would like to know what part of the task space is occupied by real baggage screening or real medical screening. In the real world, feedback is imperfect, but not entirely absent. Tasks are difficult and do produce false alarms, as in our task, but trained specialist observers, such as baggage screeners and mammographers, undoubtedly know that they have found a target on many target-present trials, as in the Fleck and Mitroff task. Moreover, the time course of individual trials is different. The robust nature of prevalence effects in the lab, even if they can be counteracted by a second response in some cases, indicates that we should determine whether they occur in the field and, if they do, how they can be counteracted.
As a topic for basic research, the differences between our results and those of Fleck and Mitroff (2007) raise a different set of interesting questions. These have to do with the problem of terminating unsuccessful searches. Changing the prevalence changes some sort of threshold for terminating search in which a target is not found. The prevalence effect shows us that the same targets that are found at high prevalence can be missed at low prevalence. It has proven difficult to model "target-absent" responses in visual search (Chun \& Wolfe, 1996; Cousineau \& Shiffrin, 2004; Zenger \& Fahle, 1997), but the prevalence data may impose constraints that clarify those models.

For the present, our results show that the prevalence effect is not always eliminated when observers have a chance to correct themselves-at least, not when the task is our simulated baggage-search task. Eliminating feedback does appear to reduce the impact of prevalence on task performance. Nevertheless, it remains clear that very low target prevalence exerts pressure on human observers. That pressure may be pernicious in tasks where miss errors are particularly undesirable. Thus, it remains worthwhile to understand the role of target prevalence in laboratory and real-world search tasks.

\section{AUTHOR NOTE}

The work reported here was funded by the Department of Homeland Security's Transportation Security Laboratory Human Factors Program, Grant DHS 02-G-010 to J.M.W. We thank Karla Evans for useful suggestions. Correspondence concerning this article should be addressed to J. M. Wolfe, Visual Attention Lab, Brigham and Women's Hospital, 64 Sidney St., Ste. 170, Cambridge, MA 02139 (e-mail: wolfe@search .bwh.harvard.edu).

\section{REFERENCES}

Baddeley, A. D., \& Colquhoun, W. P. (1969). Signal probability and vigilance: A reappraisal of the "signal-rate" effect. British Journal of Psychology, 60, 169-178.

Brainard, D. H. (1997). The Psychophysics Toolbox. Spatial Vision, 10, 433-436.

Chun, M. M., \& Wolfe, J. M. (1996). Just say no: How are visual searches terminated when there is no target present? Cognitive Psychology, 30, 39-78

Colquhoun, W. P., \& Baddeley, A. D. (1967). Influence of signal probability during pretraining on vigilance decrement. Journal of Experimental Psychology, 73, 153-155.

Cousineau, D., \& Shiffrin, R. M. (2004). Termination of a visual search with large display size effects. Spatial Vision, 17, 327-352.

Donaldson, W. (1992). Measuring recognition memory. Journal of Experimental Psychology: General, 121, 275-277.

FLECK, M. S., \& MitrofF, S. R. (2007). Rare targets are rarely missed in correctable search. Psychological Science, 18, 943-947.

GREEN, D. M., \& SwETs, J. A. (1966). Signal detection theory and psychophysics. New York: Wiley.

Gur, D., Sumkin, J. H., Rockette, H. E., Ganott, M., Hakim, C., Hardesty, L., ET AL. (2004). Changes in breast cancer detection and mammography recall rates after the introduction of a computer-aided detection system. Journal of the National Cancer Institute, 96, 185190.

Healy, A. F., \& Kubovy, M. (1981). Probability matching and the formation of conservative decision rules in a numerical analog of signal detection. Journal of Experimental Psychology: Human Learning \& Memory, 7, 344-354.

Jiang, Y., Miglioretti, D. L., Metz, C. E., \& Schmidt, R. A. (2007). Breast cancer detection rate: Designing imaging trials to demonstrate improvements. Radiology, 243, 360-367. 
Li, H., Li, F., Gao, H. H., Chen, A., \& Lin, C. (2006). Appropriate responding can reduce miss errors in visual search. Unpublished manuscript.

MackWorth, J. F., \& TAYlor, M. M. (1963). The $d^{\prime}$ measure of signal detectability in vigilance-like situations. Canadian Journal of Psychology, 17, 302-325.

Macmillan, N. A., \& Creelman, C. D. (2005). Detection theory: A user's guide (2nd ed.). Mahwah, NJ: Erlbaum.

MadDox, W. T. (2002). Toward a unified theory of decision criterion learning in perceptual categorization. Journal of the Experimental Analysis of Behavior, 78, 567-595.

Palmer, J., Verghese, P., \& Pavel, M. (2000). The psychophysics of visual search. Vision Research, 40, 1227-1268.

Pastore, R. E., Crawley, E. J., Berens, M. S., \& Skelly, M. A. (2003). "Nonparametric" $A$ ' and other modern misconceptions about signal detection theory. Psychonomic Bulletin \& Review, 10, 556-569.

Pelli, D. G. (1997). The VideoToolbox software for visual psychophysics: Transforming numbers into movies. Spatial Vision, 10, 437-442.

Pisano, E. D., Gatsonis, C., Hendrick, E., Yaffe, M., Baum, J. K., ACHARYYA, S., ET AL. (2005). Diagnostic performance of digital versus film mammography for breast-cancer screening. New England Journal of Medicine, 353, 1773-1783.
Rich, A. N., Kunar, M. A., Van Wert, M. J., Hidalgo-Sotelo, B., Horowitz, T. S., \& Wolfe, J. M. (2008). Why do we miss rare targets? Exploring the boundaries of the low prevalence effect. Journal of Vision, 8(15, Art. 15), 1-17.

RUBENSTEIN, J. (2001). Test and evaluation plan: X-Ray Image Screener Selection Test (No. DOT/FAA/AR-01/47). Washington, DC: Office of Aviation Research.

Smith, P. A., \& Turnbull, L. S. (1997). Small cell and "pale" dyskaryosis. Cytopathology, 8, 3-8.

Wolfe, J. M., Horowitz, T. S., \& Kenner, N. M. (2005). Rare items often missed in visual searches. Nature, 435, 439-440.

Wolfe, J. M., Horowitz, T. S., Van Wert, M. J., Kenner, N. M., Place, S. S., \& KibBi, N. (2007). Low target prevalence is a stubborn source of errors in visual search tasks. Journal of Experimental Psychology: General, 136, 623-638.

Zenger, B., \& FAHLE, M. (1997). Missed targets are more frequent than false alarms: A model for error rates in visual search. Journal of Experimental Psychology: Human Perception \& Performance, 23, 1783-1791.

(Manuscript received January 4, 2008;

revision accepted for publication October 4, 2008.) 